\title{
Logarithmic corrections to correlation decay in two-dimensional random-bond Ising systems
}

\author{
Jean C. Lessa* \\ Departamento de Física, Universidade Estadual de Feira de Santana, \\ Campus Universitário, 44031-460 Feira de Santana BA, Brazil \\ S.L.A. de Queiro讯 \\ Instituto de Física, Universidade Federal do Rio de Janeiro, \\ Caixa Postal 68528, 21941-972 Rio de Janeiro RJ, Brazil
}

(Dated: 28th October 2018)

\begin{abstract}
The statistics of critical spin-spin correlation functions in Ising systems with non-frustrated disorder are investigated on a strip geometry, via numerical transfer-matrix techniques. Conformal invariance concepts are used, in order to test for logarithmic corrections to pure power-law decay against distance. Fits of our data to conformal-invariance expressions, specific to logarithmic corrections to correlations on strips, give results with the correct sign, for the moments of order $n=0-4$ of the correlation-function distribution. We find an interval of disorder strength along which corrections to pure-system behavior can be decomposed into the product of a known $n$-dependent factor and an approximately $n$-independent one, in accordance with predictions. A phenomenological fitting procedure is proposed, which takes partial account of subdominant terms of correlation-function decay on strips. In the low-disorder limit, it gives results in fairly good agreement with theoretical predictions, provided that an additional assumption is made.

PACS numbers: PACS numbers: 05.50.+q, 75.10.-b
\end{abstract}

\section{INTRODUCTION}

Magnetic systems with quenched disorder exhibit properties which often differ from those of their homogeneous counterparts. The Harris criterion 1] for the relevance of disorder, with respect to possible changes of universality class at the magnetic phase transition, boils down to the question of whether the pure-system specific heat exponent $\alpha$ is positive or negative. In the former case, the universality class changes, relative to that of the pure system, while in the latter it does not. For the two-dimensional Ising model, the Harris criterion is indecisive, as the pure-system specific heat diverges logarithmically. Thus, alternative schemes must be formulated in order to investigate this borderline case. Nowadays, mounting evidence [2, 3] suggests that the critical behavior of two-dimensional Ising ferromagnets is only slightly modified by the introduction of non-frustrated disorder. The corresponding changes are given by logarithmic corrections to pure-system power-law singularities. Though the theoretical framework underlying such corrections is well-understood, their detection in numerical studies turns out to be a subtle matter [4, 5, 6, 7].

Upon introduction of disorder, the scaling dimensions associated to the moments of correlation functions at criticality acquire multifractal behavior [8], characterized by a set of independent exponents, as opposed to the pure case where a single exponent is expected.

\footnotetext{
*Electronic address: jean@if.ufrj.br
}

${ }^{\dagger}$ Electronic address: sldq@if.ufrj.br
It was analytically predicted by Ludwig [9] that the moments of the spin-spin correlation functions of the disordered Ising model behave asymptotically as:

$$
\left[\left\langle\sigma_{0} \sigma_{R}\right\rangle_{J_{i j}}^{n}\right] \sim R^{-n / 4}(\ln R)^{n(n-1) / 8}
$$

where $n=1,2, \ldots$, and the label $J_{i j}$ denotes average over quenched disorder (bond) configurations. The power-law term $R^{-n / 4}$ corresponds to the uniform-system correlation decay, while the logarithmic corrections are given by $(\ln R)^{n(n-1) / 8}$. Critical correlation functions are non-self averaging quantities, that is, the width of their distribution stays essentially constant as the number of samples $N$ increases; however, the sample-to-sample fluctuations of averaged values (e.g., the assorted moments just mentioned) do go down approximately with the square root of sample size as the latter increases [10].

For the two-dimensional Ising model, the "typical", or most probable, correlation function, defined for a specific disorder configuration (i.e., the zeroth order moment of the distribution), is predicted [9] to behave as:

$$
G_{0} \equiv \exp [\ln G(R)]_{\mathrm{av}} \sim R^{-1 / 4}(\ln R)^{-1 / 8},
$$

where, again, the uniform-system behavior $(G(R) \sim$ $\left.R^{-\eta}, \eta=1 / 4\right)$ is accompanied by a logarithmic correction. Note that corrections are expected to be absent for $n=1$, i.e., for the average correlation decay. For higher moments $n \geq 2$, the correction terms should be present, with corresponding exponents $\lambda_{n} \equiv n(n-1) / 8$, given in Eq. (1).

Here we apply numerical transfer-matrix methods, together with finite-size scaling and conformal invariance concepts, to investigate the random-bond Ising model on 
strips of a square lattice $(L \times N, N \rightarrow \infty)$. Our purpose is to confirm the analytical results derived by Ludwig, for the exponents $\eta_{n}=n / 4$ and $\lambda_{n}=n(n-1) / 8$, associated to the moments of critical correlation functions of the model.

\section{METHOD}

We consider a random-bond Ising system on a square lattice, whose Hamiltonian is given by

$$
\mathscr{H}=-\sum_{i, j} J_{i j} \sigma_{i} \sigma_{j}
$$

where $\sigma_{i}= \pm 1$ are the (site) spin variables, and $J_{i j}$ are ferromagnetic interactions between nearest-neighbor spins, extracted from the quenched probability distribution:

$$
P\left(J_{i j}\right)=\frac{1}{2}\left[\delta\left(J_{i j}-J_{0}\right)+\delta\left(J_{i j}-r J_{0}\right)\right], 0 \leqslant r \leqslant 1 .
$$

The exact critical temperature $\beta_{c} \equiv 1 / k_{B} T_{c}$ is known from duality [11, 12]

$$
\sinh \left(2 \beta_{c} J_{0}\right) \sinh \left(2 \beta_{c} r J_{0}\right)=1 .
$$

In our calculations we initially consider a system with $r=$ $1 / 4$, for which Eq. (5) gives $T_{c}(1 / 4) / J_{0}=1.239 \cdots$ (in these units, the critical point of the uniform system is at $\left.T_{c}(1) / J_{0}=2.269 \cdots\right)$. We shall also investigate stronger disorder, by using $r=0.1,0.05$, and 0.01 (for the latter, the critical temperature is $\left.T_{c}(0.01) / J_{0}=0.5089 \cdots\right)$. The calculation of spin-spin correlation functions follows the lines of Section 1.4 of Ref. 13, with standard adaptations for an inhomogeneous system[14]. Taking two spins on, say, row 1 , separated by a distance $R$, and for a given configuration $\mathcal{C}$ of bonds, one has:

$$
\left\langle\sigma_{0}^{1} \sigma_{R}^{1}\right\rangle_{\mathcal{C}}=\frac{\sum_{\sigma_{0} \sigma_{R}} \tilde{\psi}\left(\sigma_{0}\right) \sigma_{0}^{1}\left(\prod_{i=0}^{R-1} \mathcal{T}_{i}\right)_{\sigma_{0} \sigma_{R}} \sigma_{R}^{1} \psi\left(\sigma_{R}\right)}{\sum_{\sigma_{0} \sigma_{R}} \tilde{\psi}\left(\sigma_{0}\right)\left(\prod_{i=0}^{R-1} \mathcal{T}_{i}\right)_{\sigma_{0} \sigma_{R}} \psi\left(\sigma_{R}\right)},
$$

where $\sigma_{0} \equiv\left\{\sigma_{0}^{1} \ldots \sigma_{0}^{L}\right\}$ and correspondingly for $\sigma_{R}$; the bonds that make the transfer matrices $\mathcal{T}_{i}$ belong to $\mathcal{C}$. For pure systems the $2^{L}$-component vectors $\tilde{\psi}, \psi$ are determined by the boundary conditions; for example, the choice of dominant left and right eigenvectors gives the correlation function in an infinite system[13]. Here, one needs only be concerned with avoiding start-up effects, since there is no convergence of iterated vectors. This is done by discarding the first few hundred iterates of the initial vector $\mathbf{v}_{0}$. From then on, one can shift the dummy origin of Eq. (6) along the strip, taking $\tilde{\psi}(\psi)$ to be the left- (right-) iterate of $\mathbf{v}_{0}^{T}\left(\mathbf{v}_{0}\right)$ up to the shifted origin. In order to avoid spurious correlations between the dynamical variables, the respective iterations of $\tilde{\psi}$ and $\psi$ must use distinct realizations of the bond distribution.
As regards conformal invariance, this has been a fundamental tool to the understanding of the critical behavior of pure systems in two dimensions. Specifically, the spin-spin correlation function on a strip can be obtained from the conformal mapping $w=x+i y=(L / 2 \pi) \ln z$, of the plane $z=u+i v$ onto a strip of width $L$ with periodic boundary conditions across, leading to the following asymptotic behavior at criticality [15]:

$$
G_{x y}^{\text {pure }} \sim\left[\frac{\pi / L}{\left(\sinh ^{2}(\pi x / L)+\sin ^{2}(\pi y / L)\right)^{1 / 2}}\right]^{\eta},
$$

where $\eta=1 / 4$ for pure Ising spins.

For disordered spin systems at criticality, one expects conformal invariance to be preserved when averages over disorder are taken. Indeed, numerical evidence for this has been found in several instances, e.g., random-bond Ising model [4, 10, 14], random-bond $q$-state Potts models [16], random transverse Ising chains at $T=0$ (equivalent to the two-dimensional McCoy-Wu model) [17], and Ising spin glasses 18, 19, 20, 21, 22.

We first recall the simpler case where correlation decay in the bulk disordered system is purely polynomial. Considering correlation functions on a strip, preservation of conformal invariance in the sense just defined means that the decay-of-correlation exponents $\eta_{i}$, associated to the assorted moments $m_{i}$ of the probability distribution (PDF) of correlation functions in the disordered magnet, can be determined via an extension of Eq. (7), i.e. by assuming:

$$
m_{i} \sim z^{-\eta_{i}}, z \equiv\left(\sinh ^{2}(\pi x / L)+\sin ^{2}(\pi y / L)\right)^{1 / 2} .
$$

The $\eta_{i}$ can be extracted by fitting numerical data to Eq. (8). Thus far, it has been found that results derived in this way (e.g. for spin glasses) are consistent with those coming from different analytical methods which do not explicitly invoke conformal invariance [18, 21, 22].

Before going further, one must recall that an extensive analysis of the inaccuracies associated to the finite strip width $L$, carried out in Ref. 21, indicates that finite-width effects are essentially subsumed in the explicit $L$ dependence given in Eq. (7). Thus, higher-order finite-size corrections presumably do not play a significant role in the conformal-invariance properties of assorted moments of the correlation-function PDF (at least when critical correlation decay in the bulk is purely power law-like).

Turning now to the present case, one must envisage how the multiplicative logarithmic corrections, predicted in Eqs. (11) and (2) for bulk correlations, translate onto a strip geometry. According to Refs. 23, 24, for distances $R \gg L$ along the strip the correlation decay should be exponential, with $m_{n} \equiv G_{n}(R) \sim \exp \left(-R / \xi_{n}\right)$, where

$$
\frac{L}{\pi \xi_{n}}=\eta_{n}-\lambda_{n} \pi b g(\ln L)+\mathcal{O}\left(g^{2}\right) \text {. }
$$

In the above, $b$ is a universal amplitude related to the 
normalization of the three-point function [23, 24], and

$$
g(\ln L)=\frac{g_{0}}{1+\pi b g_{0} \ln L},
$$

where $g_{0}$ is proportional to the intensity of disorder. In the limit $L \rightarrow \infty$, Eq. (9) turns into a $g_{0}$-independent form,

$$
\frac{L}{\pi \xi_{n}}=\eta_{n}-\frac{\lambda_{n}}{\ln L}+\mathcal{O}\left(\frac{1}{\ln L}\right)^{2},
$$

A semi-quantitative verification of Eq. (11) was given, for $n=0$ only, in Ref. 4 .

In Sec. III we at first apply Eq. (8) to our data, i.e. logarithmic corrections are not explicitly considered. The idea is to keep as close as possible to the framework in which conformal invariance is known to apply to disordered systems, and check whether the resulting effective exponents, $\eta_{i}^{\text {eff }}$, deviate from their pure-system values in a way consistent with the corrections predicted in Eqs. (11) and. (2). Then we attempt to extract quantitative information, by comparing our numerical results to Eq. (9), as well as its asymptotic form, Eq. (11). Finally, we investigate a phenomenological scheme to incorporate subdominant corrections to exponential correlation decay on a strip, which is subsequently applied to the analysis of low-disorder data.

\section{NUMERICAL RESULTS}

Estimation of uncertainties associated to the several moments of the correlation-function PDFs follows the procedure described in Ref. 10, where it was shown that for finite-width strips of length $N$, the width of the PDF remains constant as $N$ varies. Though the dispersions $\Delta G$ (or $\Delta(\ln G))$ do not shrink down to zero, it is possible to extract reliable information about average values, as the dispersion of these among independent samples is proportional to $1 / \sqrt{N}$. One can check, for instance, that fluctuations of said averages are $\lesssim 1 \%$ for a strip of length $N=10^{6}$.

In Fig. 1 we present the moments $m_{n}$ of the correlation-function PDF against the variable $z$, where we used $L=16$ and $N=10^{5}$ for $1 \leq x \leq 5,0 \leq y \leq 8$. From least-squares fits of the data, one can find estimates for the effective exponents $\eta_{i}^{\text {eff }}$ in the form suggested by Eq. (8). Results are displayed in Table I Deviations from pure-system values are consistent with the signs predicted for logarithmic corrections from Eqs. (11) and (2), namely an enhancement for $n=0$, approximate neutrality for $n=1$, and reduction for $n \geq 2$.

In order to produce a more quantitative analysis, we now resort to Eqs. (9), (11).

In all cases to be described below, we used $L=10$, and the horizontal distance $x$ was taken long enough in order to try and detect the effects which give rise to logarithmic corrections [23, 24], see Eqs. (9), (11). In practice,

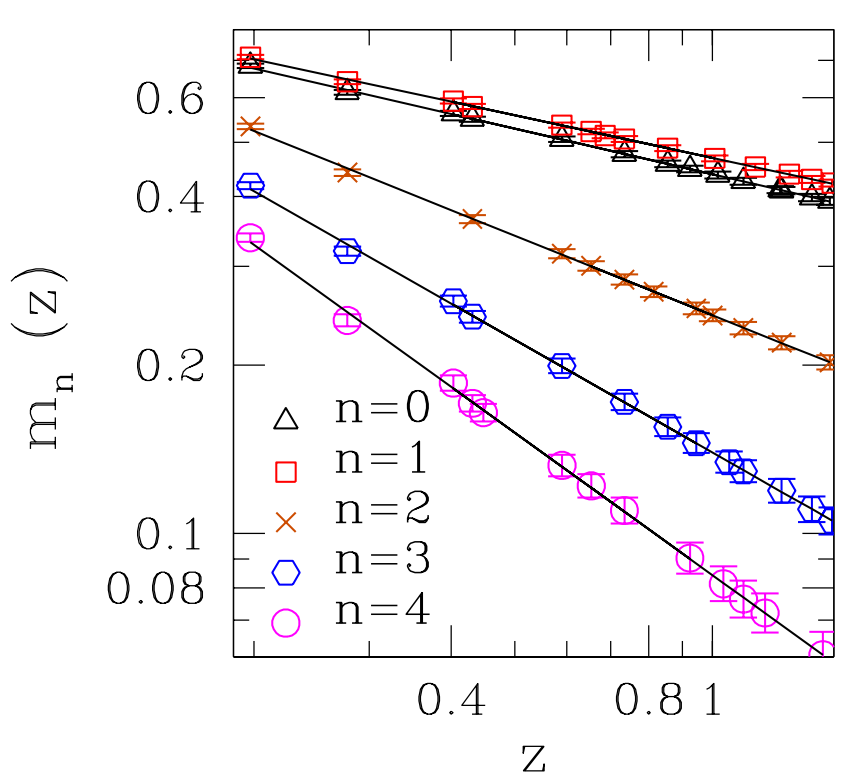

Figure 1: (Color online) Double-logarithmic plot of moments of the correlation-function distribution, against $z=$ $\left(\sinh ^{2}(\pi x / L)+\sin ^{2}(\pi y / L)\right)^{1 / 2}$, for $r=1 / 4, L=16, N=$ $10^{5}$. Lines are least-squares fits of single power-law form to data, from whose slopes one extracts estimates for the $\eta_{n}^{\text {eff }}$ of Eq. (8).

Table I: Estimates of effective exponents $\eta_{i}^{\text {eff }}$, from leastsquares fits of moments of correlation-function distributions to the form $m_{i} \sim z^{-\eta_{i}^{\text {eff }}}$. Data for $r=1 / 4, L=16$ and $N=10^{5}$.

\begin{tabular}{ll}
\hline \hline$i$ & $\eta_{i}^{\text {eff }}$ \\
\hline 0 & $0.271(2)$ \\
1 & $0.252(2)$ \\
2 & $0.473(4)$ \\
3 & $0.671(8)$ \\
4 & $0.85(1)$ \\
\hline \hline
\end{tabular}

we used $12 \lesssim x \lesssim 35$ lattice spacings along the strip, as it was noticed that the use of larger $x$ gave rise to large sample-to-sample fluctuations, thus compromising the accuracy of our data. Also, and for the sake of simplicity, we made $y=0$.

Here we evaluated 10 independent estimates for $N=$ $10^{5}$ for the moments of the correlation-function PDF, and took error bars as three times the standard deviation among these. This was necessary, since it is these uncertainties which constitute the single major impediment to the accurate estimation of the exponents $\eta_{n}$ and $\lambda_{n}$.

In the semi-logarithmic plot of Fig. 2, one can see that 


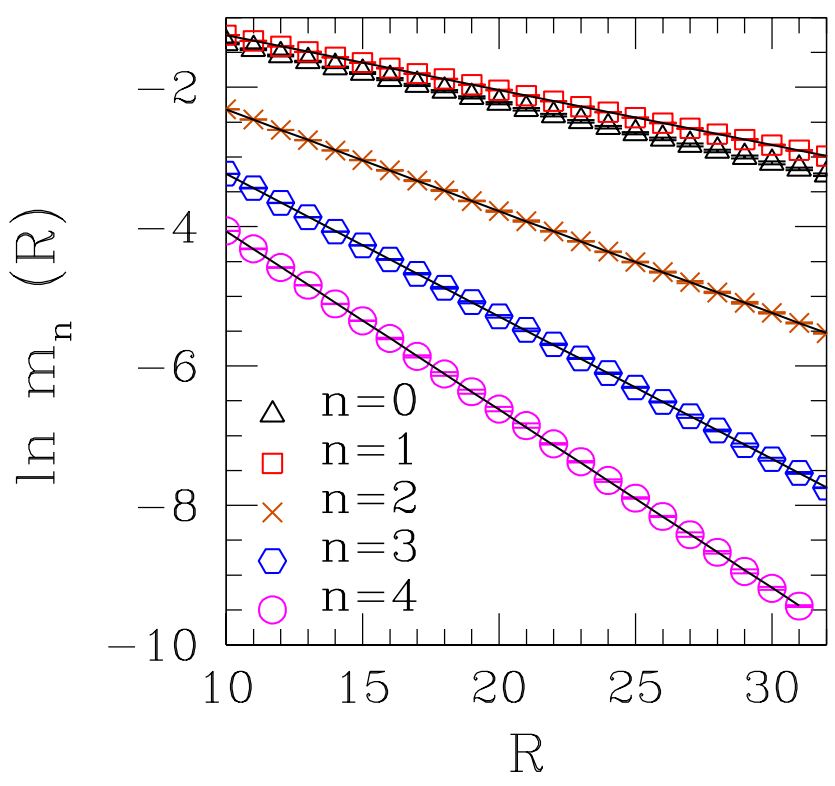

Figure 2: (Color online) Semi-logarithmic plot of moments of the correlation-function distribution, against distance $R$ along the strip (i.e. making $y=0$ in Eq. (7)). Straight lines are least-squares fits of data, from whose slopes the assorted values of $\lambda_{n}$ in Table II have been estimated (see text). Here $r=1 / 4, L=10, N=10^{5}$.

Table II: Estimates of exponents $\lambda_{n}$, from least-squares fits of moments of correlation-function distributions to a pureexponential decay form, as given by Eq. (11), keeping the $\eta_{n}$ fixed at their pure-system values: $\eta_{0}=1 / 4, \eta_{n}=n / 4(n \geq 1)$. Data for $r=1 / 4, L=10$ and $N=10^{5}$. Uncertainties in last quoted digits are shown in parentheses.

\begin{tabular}{ccc}
\hline \hline$n$ & $\lambda_{n}$ (calc.) & $\lambda_{n}$ (expected) \\
\hline 0 & $-0.05(1)$ & $-1 / 8$ \\
1 & $-0.0012(7)$ & 0 \\
2 & $0.081(1)$ & $1 / 4$ \\
3 & $0.218(2)$ & $3 / 4$ \\
4 & $0.405(4)$ & $3 / 2$ \\
\hline \hline
\end{tabular}

an exponential decay with distance, which is a precondition for Eqs. (9), (11) to apply, has set in to a good degree of accuracy for the range of $R$ depicted. We first checked whether the values of strip width $L=10$, and disorder $r=1 / 4$ used so far, might correspond to the asymptotic regime described by Eq. (11). The corresponding estimates for $\lambda_{n}$, shown in Table from least-squares fits of our data to the form given in Eq. (11), by keeping the $\eta_{n}$ fixed at their pure-system values: $\eta_{0}=1 / 4, \eta_{n}=n / 4(n \geq 1)$.

As can be seen, though the estimates extracted from our data have their sign as predicted, their magnitude is at most only about one-third of the expected (except for $n=1$, in which case the calculated result may be taken as broadly consistent with the predicted absence of corrections). The most likely explanation for such discrepancy is, therefore, that the asymptotic regime has not been reached yet. Similar considerations were used in Ref. 23, where the 4-state Potts model was analysed with help of the generic (i. e., non-asymptotic) form, Eq. (9). From free-energy data, the numerical values of $g(\ln L)$ for $L=6-10$ were extracted, and it was shown that they explained the respective deviations of gap scaling data from their known exact values.

A major obstacle to following the same steps here is the presence of randomness, which severely limits the accuracy of both free-energy (see, e g., Ref. 14) and correlation-function estimates. Furthermore, the exact bulk free energy is not known, contrary to the case of the 4-state Potts model [23].

We then compared correlation-function data pertaining to different values of $L$, namely $L=10$ and 16 (both for $r=1 / 4$ ). We found that, e. g., fitting $L=16$ results to the asymptotic form Eq. (11) gives estimates for $\lambda_{n}$ which fall essentially within the error bars of those given in Table I for $L=10$. In addition to the randomness effects just mentioned, such poor resolution also reflects the logarithmic $L$-dependence of the corrections under study, and indicates that for the values of $L \lesssim 25$ within current reach of transfer-matrix calculations, there is little hope of extracting reliable information from varying $L$.

Next, we investigated the behavior of our results against varying disorder. This means varying $r$ in Eq. (4), which corresponds to changing $g_{0}$ in Eq. (10). The actual functional dependence $g_{0}=g_{0}(r)$ is not known, apart from the basic fact that $g_{0}$ must increase as $r$ decreases towards zero. A similar degree of freedom is absent in the 4-state Potts model investigated in Ref. 23. From Eqs. (9), (10), and (11), by doing this for fixed $L$ one expects to move along the crossover between small- and large $-L$ regimes.

We calculated moments of order $0-4$ of the correlationfunction PDFs, for $r=0.1,0.05$, and 0.01. Fits of our data to the asymptotic form, Eq. (11), are shown in Fig. 3. For each $n \neq 1$, the sign of calculated exponents remains in agreement with predictions and their absolute value increases with increasing disorder $\left(\lambda_{1}\right.$ remains close to zero, reaching 0.07(1) for $r=0.01$ ). However, the trend against increasing disorder is clearly towards overshooting the theoretical predictions, with no sign of stabilization.

It seems reasonable to exclude the possibility of nonmonotonic behavior for even smaller $r$, which would then bring the estimated exponents back to their predicted values. The simplest explanation for the behavior depicted in Fig. 3 is that, at $r \lesssim 0.05$ or thereabouts (for fixed $L=10$ ), the effects of the percolation fixed point at $r=0, T_{c}(0)=0$, are beginning to manifest themselves. Indeed, the description synthesized in Eq. (9) implicitly 


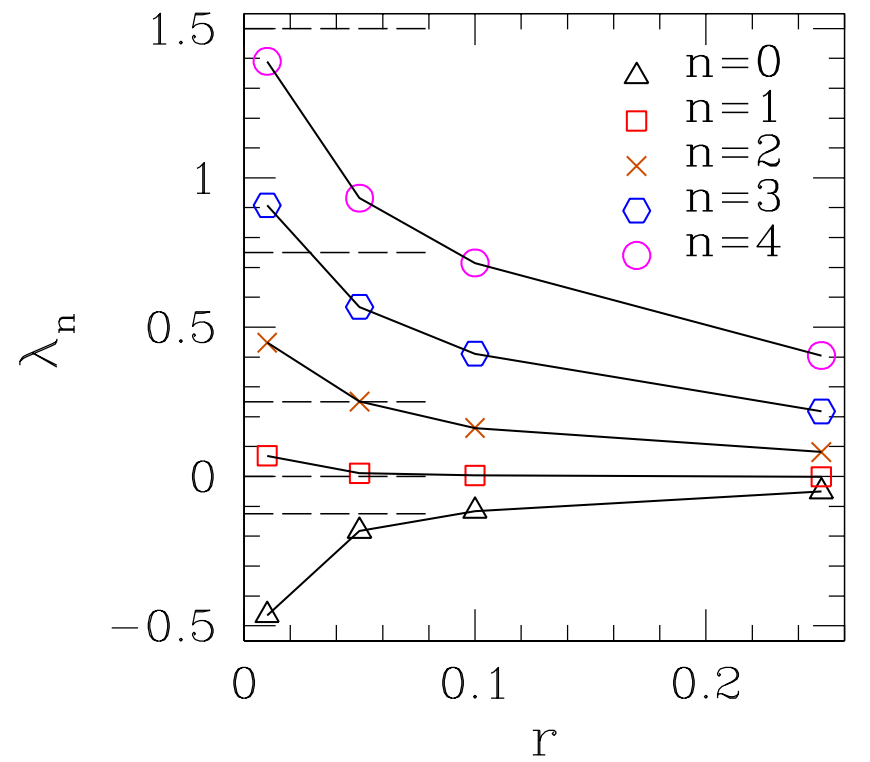

Figure 3: (Color online) Logarithmic correction exponents $\lambda_{n}$, calculated via the asymptotic expression, Eq. (11) from moments of the correlation-function distribution, against disorder intensity $r$ (disorder increases with decreasing $r$, see Eq. (4)). Error bars are of the same order of, or smaller than, symbol sizes. Dashed horizontal lines starting on vertical axis mark expected values of $\lambda_{n}=-1 / 8,0,1 / 4,3 / 4$, and $3 / 2$ respectively for $n=0-4$. All for $L=10, N=10^{5}$.

envisages a perturbation scenario where pure-Ising critical behavior is modified by a marginally-relevant operator, which induces the logarithmic corrections investigated here. Thus it is plausible that such framework would fail to include the extreme disorder close to the zero-temperature fixed point.

In order to check this hypothesis, we must go back to the non-asymptotic form, Eq. (9), and ask whether (for fixed $L$ ) there is a range of disorder in which the corrections to the pure-system exponents can be decomposed into the product of an $n$-dependent and an $n$-independent factor (respectively, $\lambda_{n}$ and $\pi b g(\ln L)$ ) as in Eq. (9), and whether such range is limited by increasing disorder. As pointed out above, in contrast with Ref. 23 here we are allowed to vary $g_{0}$, which can then substitute for the $L$ variation investigated in that Reference.

We assumed the $\eta_{n}$ and $\lambda_{n}$ to have their exact predicted values and, from the numerical estimates of moments of correlation-function PDFs, calculated $\pi b g(\ln L)$ for $n=0,2,3$, and 4 . The results are exhibited in Fig. 4. We first note that the $n=0$ data show a much wider range of variation than the rest. This may be related to specific properties of the zeroth moment of the correlation-function PDF, in whose calculation the occurrence of small values plays a disproportionately important role (compared to the moments of order $n \geq 1$ ).



Figure 4: (Color online) Estimates of $\pi b g(\ln L)$ of Eq. (11) (assuming $\eta_{n}, \lambda_{n}$ to have their exact values) calculated from moments of the correlation-function distribution, against disorder intensity $r$. Error bars are of the same order of, or smaller than, symbol sizes. All for $L=10, N=10^{5}$.

However, we cannot advance a specific explanation at this point. We decided to concentrate on the analysis of $n=2-4$ data, whose average gives $\pi b g(\ln L)=0.30(3)$ for $r=1 / 4$, and $0.56(8)$ for $r=0.1$. For smaller $r$ we see that the scatter becomes larger, in a way consistent with the idea that the physical picture of Eq. (9) becomes frayed as disorder increases. The agreement is qualitative and, to a fair extent, quantitative, inasmuch as both Figures 3 and 4 concur in indicating $r \approx 0.1$ as the threshold beyond which the perturbative view definitely fails (for $L \approx 10$, that is; for larger $L$ such threshold would presumably move towards higher disorder).

We now return to the relatively low-disorder case of $r=1 / 4$, and investigate whether a perturbative scheme exists, which may account for the features exhibited by correlation functions on a strip geometry. Specifically, Eq. (9) predicts that, in the disordered system, correlations on a strip still decay purely exponentially, as in pure-Ising strips, only with a small change in the exponent. In the crossover regime to which $L=10, r=1 / 4$ certainly belong, such incipient modification may turn up as an effective subdominant (i.e. less than exponential) correction to our numerics. Thus, one could expect an effective behavior:

$$
G_{n}(R) \sim \exp \left(-\pi R \eta_{n} / L\right)[R]^{\Lambda_{n}},
$$

where $\eta_{n}$ are the pure-system exponents, and subdominant corrrections are assumed to take the simplest pos- 
sible form of a power law, with the $\Lambda_{n}$ to be determined. Following this reasoning, and recalling the behavior of bulk correlations invoked in Eqs. (11) and (2), one sees that a literal transposition of the $R$-dependence of the latter onto the $z$-dependence of strip correlations (in analogy with Eq. (8)), would mean:

$$
G_{n}(z) \sim z^{-\eta_{n}}[\ln z]^{\lambda_{n}}=\left[z(\ln z)^{q_{n}}\right]^{-\eta_{n}},
$$

where $\eta_{0}=1 / 4, \lambda_{0}=-1 / 8, \eta_{n}=n / 4, \lambda_{n}=n(n-$ $1) / 8(n \geq 1)$, and $q_{n}=-\lambda_{n} / \eta_{n}$. In the regime $x=$ $R \gg L, y=0$ considered in Eq. (9), which corresponds to $z \sim \exp (\pi R / L)$ in Eq. (8), this indeed amounts to writing

$$
G_{n}(R) \sim \exp \left(-\pi R \eta_{n} / L\right)[R]^{\lambda_{n}},
$$

i.e., pure-Ising exponential decay with power-law corrections. Since Eq. (13) does not, as far as we are aware, have a rigorous justification on the basis of conformal invariance, the predicted identification $\Lambda_{n}=\lambda_{n}$ extracted from comparison of Eq. (12) to Eq. (14) must be regarded warily. Nevertheless, from the preceding analysis summarized in Tables \and II for each $n$ one expects $\Lambda_{n}$ and $\lambda_{n}$ to have the same sign and, possibly, to be of similar orders of magnitude. Therefore, in what follows we shall take considerations based on Eq. (13) as a starting point to reanalyze the set of data displayed in Fig. 2]

Using Eq. (13), the moment of order $n=0$ must correspond to $q=1 / 2$. In Fig. 5 . fitting data for the moment of order $n=0$ we at first fixed $q=1 / 2$ (as suggested by Eq. (2) ), and allowed $\eta_{0}$ to vary in Eq. (13). The best fit was found for $\eta_{0}=0.2505(7)$, corresponding to a $\chi^{2}$ per degree of freedom ( $\left.\chi_{\text {d.o.f. }}^{2}\right)$ equal to 0.012 . We then fixed $\eta_{0}$ at the central estimate just found, and allowed $\Lambda_{0}$ of Eq. (12) to vary. The $\chi_{\text {d.o.f. for this latter fit are }}^{2}$ displayed in the insert of Fig. [5] with a clear minimum at $\Lambda_{0}=-0.125$.

According to Eq. (11), the moment of order $n=1$ is not expected to present logarithmic corrections. In order to check this, we fixed $q=0$ in Eq. (13) and allowed $\eta_{1}$ to vary. The best fit, shown in Fig. 6] was found for $\eta_{1}=0.2505(1)$, very close to $1 / 4$ of a uniform system, corresponding to a $\chi_{\text {d.o.f. }}^{2}=0.02$. In the insert of the Figure, one can see that, upon keeping $\eta_{1}$ fixed at the central estimate quoted above, and allowing $\Lambda_{1}$ of Eq. (12) to vary, the closest fit indeed occurs for $\Lambda_{1}=0$.

When turning to higher-order moments $n \geq 2$, we noticed that it was not possible, in general, to produce good fits of the whole range of data displayed in Fig. 2 to the variables suggested by Eq. (13). However, subsets of the data did fit well against such variables, as is illustrated in Fig. 17. We shall return to this point at the end of the Section.

For the moment of order $n=2$, again we fixed $q=-1 / 2$ in Eq. (13) and allowed $\eta_{2}$ to vary. The best fit was found for $\eta_{2}=0.4992(8)$, corresponding to a $\chi_{\text {d.o.f. }}^{2}=0.03$. Data for this setup are displayed in the

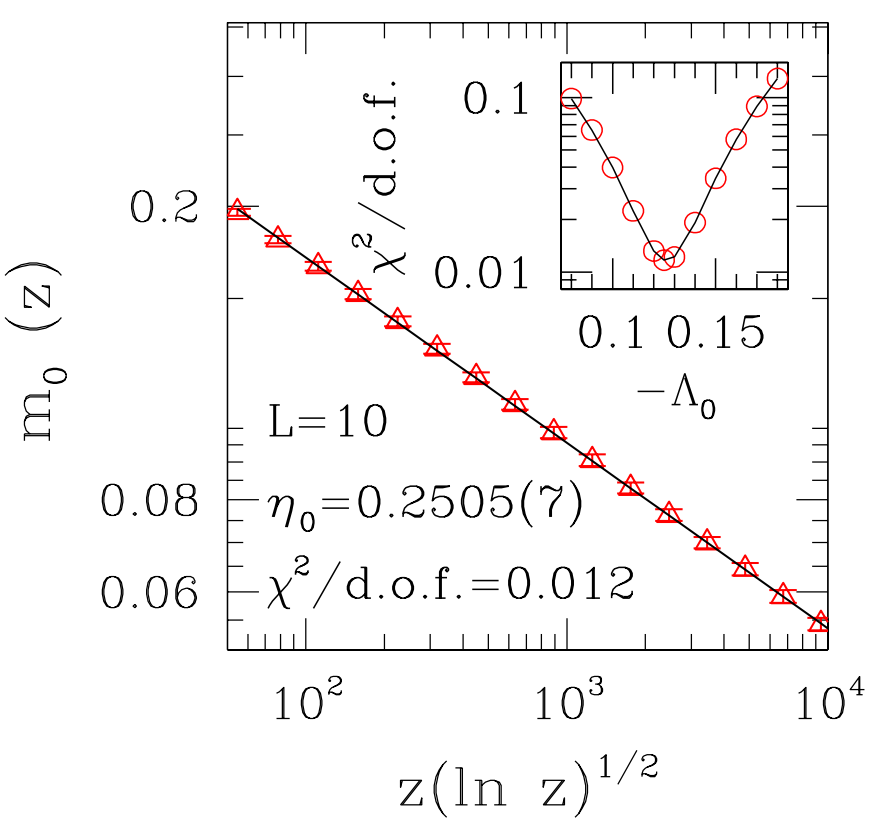

Figure 5: (Color online) Main diagram: double-logarithmic plot of moment of order $n=0$ of correlation-function $\mathrm{PDF}$, against $\left[z(\ln z)^{q}\right]$ for $q=1 / 2$. Line corresponds to $\eta_{0}=0.2505$ (best estimate obtained for fixed $q=1 / 2$, see text); $L=10, r=1 / 4$. Insert: semi-logarithmic plot of $\chi_{\text {d.o.f. against }}^{2}-\Lambda_{0}$, for fits of $n=0$ moment data against $z^{-\eta_{0}}[\ln z]^{\Lambda_{0}}$, with $\eta_{0}=0.2505$ (fixed).

Table III: Estimates of exponents $\eta_{n}$ and $\Lambda_{n}$, from leastsquares fits of moments of correlation-function distributions obtained with $G_{n}(z) \sim\left[z(\ln z)^{q_{n}}\right]^{-\eta_{n}}$, as given by Eq. (13); see also Eq. (12). Data for $r=1 / 4, L=10$ and $N=10^{5}$. Uncertainties in last quoted digits are shown in parentheses.

\begin{tabular}{ccc}
\hline \hline$n$ & $\eta_{n}$ & $\Lambda_{n}$ \\
\hline 0 & $0.2505(7)$ & $-0.124(4)$ \\
1 & $0.2505(1)$ & $0.000(2)$ \\
2 & $0.4992(8)$ & $0.248(6)$ \\
3 & $0.751(2)$ & $0.75(1)$ \\
4 & $1.032(6)$ & $1.54(4)$ \\
\hline \hline
\end{tabular}

main diagram of Fig. 8. In the insert of the Figure, we kept $\eta_{2}$ fixed at the central estimate just found, and allowed $\Lambda_{2}$ of Eq. (12) to vary. The $\chi_{\text {d.o.f. }}^{2}$ of the respective fits are shown, with a clear minimum at $\lambda_{2}=0.25$.

The previous procedure was applied for the moments of orders $n=3$ and $n=4$. The results for all investigated exponents, $\eta_{n}$ and $\Lambda_{n}$ are displayed in Table III.

It can be seen from Table III that both accuracy and precision (the latter, upon comparison with theoretical predictions of $\lambda_{n}$ ) of results become lower for higherorder moments; in particular, our estimate of $\eta_{4}$ already differs somewhat from $\eta_{4}=1$ predicted in Eq. (2) 




Figure 6: (Color online) Main diagram: double-logarithmic plot of moment of order $n=1$ of correlation-function PDF, against $\left[z(\ln z)^{q}\right]$ for $q=0$. Line corresponds to $\eta_{1}=0.2505$ (best estimate obtained for fixed $q=0$, see text); $L=10$, $r=1 / 4$. Insert: semi-logarithmic plot of $\chi_{\text {d.o.f. against }}^{2} \Lambda_{1}$ , for fits of $n=1$ moment data against $z^{-\eta_{1}}[\ln z]^{\Lambda_{1}}$, with $\eta_{1}=0.2505$ (fixed).



Figure 7: (Color online) Double-logarithmic plot of higherorder moments of correlation-function $\mathrm{PDF}$, against $\left[z(\ln z)^{q}\right]$ with $q=-1 / 2,-1$, and $-3 / 2$ respectively for $n=2,3$, and 4. Thick continuous lines show range of data used in leastsquares fits of the $\eta_{n}$ for $q$ fixed as above. $L=10, r=1 / 4$.

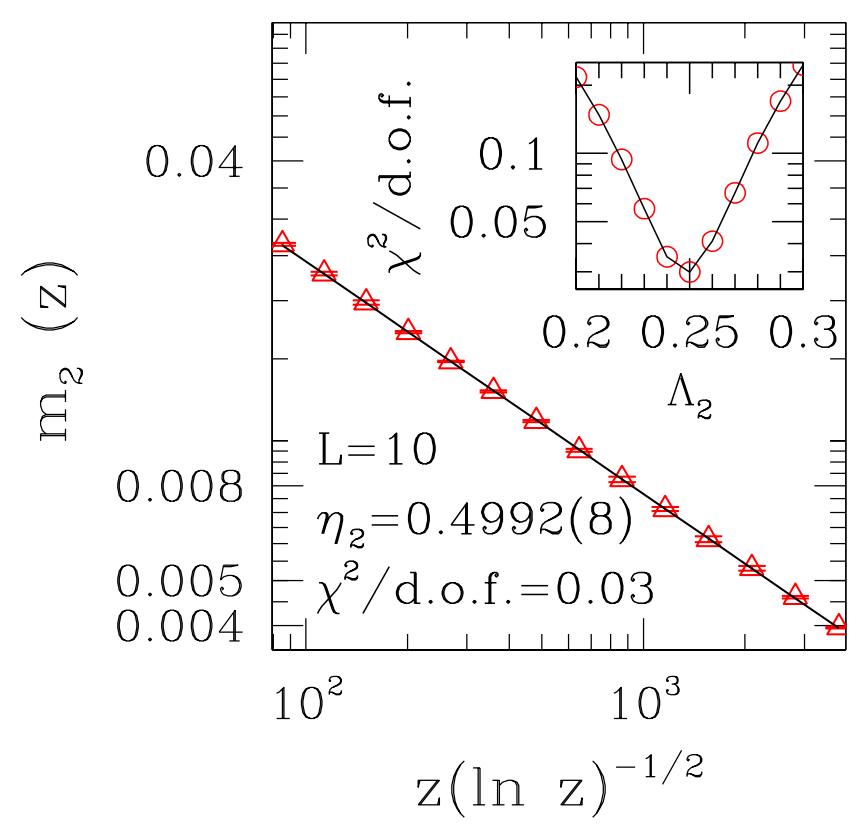

Figure 8: (Color online) Main diagram: double-logarithmic plot of moment of order $n=2$ of correlation-function PDF, against $\left[z(\ln z)^{q}\right]$ for $q=-1 / 2$. Line corresponds to $\eta_{2}=$ 0.4992 (best estimate obtained for fixed $q=-1 / 2$, see text); $L=10, r=1 / 4$. Insert: semi-logarithmic plot of $\chi_{\text {d.o.f. }}^{2}$ against $\Lambda_{2}$, for fits of $n=2$ moment data against $z^{-\eta_{2}}[\ln z]^{\Lambda_{2}}$, with $\eta_{2}=0.4992$ (fixed).

(though that for $\Lambda_{4}$ is consistent, at the margin, with $\lambda_{4}=3 / 2$ from the same Equation). For $n>4$ we have found that the situation worsens considerably. This is because: (i) short-distance deviations from asymptotic behavior tend to increase with $n$, and (ii) at the relatively long distances where asymptotic behavior sets in, the correlation functions themselves, and a fortiori their higher moments, become smaller, and their averages prone to higher relative fluctuations. We recall the trend illustrated in Figure 7 against increasing $n$ : for all three sets of data shown there, the thick continuous lines (corresponding respectively to $19 \leq x \leq 32(n=2)$, $21 \leq x \leq 32(n=3)$, and $20 \leq x \leq 31(n=4))$ denote the range of data used to extract the results quoted in Table III One sees that, since $|q|$ increases with $n$, sets of data for the same range of $x$ become more compressed when plotted against $\left[z(\ln z)^{q}\right]$, thus increasing uncertainties upon fitting.

As one might expect, such a picture of good fits to Eq: (12), producing estimates of $\eta_{n}$ and $\lambda_{n}$ quite close to the exact values, does not hold for higher disorder. For instance, already for $r=0.1, n=2$, our best fit to Eq. 12) gives $\eta_{2}=0.433(1), \lambda_{2}=0.019(5)\left(\chi_{\text {d.o.f. }}^{2}=\right.$ 0.04 ). Fixing $\eta_{2}=1 / 2, \lambda_{2}=1 / 4$ gives $\chi_{\text {d.o.f. }}^{2} \approx 24$. 


\section{CONCLUSIONS}

We have tested the theoretical predictions, given in Eqs. (1) and (2), of logarithmic corrections to the asymptotic behavior of spin-spin correlation functions, in the two-dimensional (unfrustrated) random-bond Ising model. The use of a strip geometry has been accompanied by the corresponding finite-size scaling and conformal-invariance considerations. So far the validity of the latter, when dealing with non-uniform systems, refers to disorder-averaged quantities, and has been established only in cases where critical correlation decay is purely polynomial [18, 21, 22].

From examination of short-distance data (see Fig. 1] and Table , we could only establish that the effective exponents obtained were consistent with corrections of the same sign as predicted by theory. The situation is similar to that found in Ref. 7, where the conformal mapping of the square lattice on a finite square was used, and only $n=0$ was investigated. Those authors concluded that, for the size of lattices used, they would be able only to assert that the sign of corrections was as predicted.

By considering suitably long distances along the strip, we attempted to reach the regime where the effect of logarithmic corrections on a strip geometry is expected to be more easily isolated, as predicted in Refs. 23, 24. Allowing the intensity of disorder to vary produced a picture in which one is able to identify the approximate range of validity of the results given in Refs. 23, 24, even for strips of relatively small width. This relies essentially on identifying the interval of disorder strength $r$, along which an approximately $n$-independent factor can be isolated from numerical data, in accordance with the non-asymptotic forms predicted in Eq. (9). The corresponding data are shown in Fig. 4

We also produced a phenomenological fitting scheme, expected to work at low disorder, and synthesized in Eq. (12), from which estimates of the postulated exponents $\Lambda_{n}$ were extracted. While the analogy to Eq. (8), used to establish Eqs. (13) and (14), seems short of a rigorous justification on the basis of conformal invariance, the ensuing prediction that $\Lambda_{n}=\lambda_{n}$ appears to be backed to a large extent by our numerical data for low disorder $r=1 / 4$. As expected, the validity of such a scheme does not extend far towards increasing disorder.

In summary, the evidence shown here provides a check of the validity of Eqs. (1) and (2) for disordered Ising systems, in the appropriate limit of long distances. It would be interesting to ascertain whether any deeper connection lies between the phenomenological form Eq. (12) and conformal-invariance properties.

\section{Acknowledgments}

J.C.L. thanks the Brazilian agency CAPES for partial financial support, and Departamento de Física de Sólidos, UFRJ, for making its research infrastructure available. The research of S.L.A.dQ. was partially supported by the Brazilian agencies CNPq (Grant No. 30.0003/2003-0), FAPERJ (Grant No. E26$152.195 / 2002)$, FUJB-UFRJ, and Instituto do Milênio de Nanociências-CNPq.
[1] A. B. Harris, J. Phys. C 7, 1671 (1974)

[2] B. N. Shalaev, Phys. Rep. 237129 (1994).

[3] W. Selke, L. N. Shchur, and A. L. Talapov, in Annual Reviews of Computational Physics, vol. 1 (World Scientific, Singapore, 1994), edited by D. Stauffer.

[4] S. L. A. de Queiroz, J. Phys. A 30, L443 (1997).

[5] F. D. A. Aarão Reis, S. L. A. de Queiroz, and R. R. dos Santos, Phys. Rev. B 56, 6013 (1997)

[6] D. Stauffer, F. D. A. Aarão Reis, S. L. A. de Queiroz, and R. R. dos Santos, Int. J. Mod. Phys. C 8, 1209 (1997).

[7] B. Berche and L.N. Shchur, JETP Lett. 79, 213 (2004).

[8] T. Olson and A. P. Young, Phys. Rev. B 60, 3428 (1999).

[9] A. W. W. Ludwig, Nucl. Phys. B 330, 639 (1990).

[10] S. L. A. de Queiroz and R. B. Stinchcombe, Phys. Rev. E 54, 190 (1996).

[11] R. Fisch, J. Stat. Phys. 18, 111 (1978).

[12] W. Kinzel and E. Domany, Phys. Rev. B 23, 3421 (1981).

[13] M. P. Nightingale, in Finite Size Scaling and Numerical Simulations of Statistical Systems, (World Scientific, Singapore, 1990), edited by V. Privman.

[14] S. L. A. de Queiroz, Phys. Rev. E 51, 1030 (1995).
[15] J. L. Cardy, in Phase Transitions and Critical Phenomena, Vol. 11 (Academic, New York, 1987), edited by C. Domb and J. L. Lebowitz.

[16] B. Berche and C. Chatelain, in Order, Disorder, and Criticality (World Scientific, Singapore, 2004), edited by Yu. Holovatch.

[17] F. Iglói and H. Rieger, Phys. Rev. Lett. 78, 2473 (1997).

[18] A. Honecker, M. Picco, and P. Pujol, Phys. Rev. Lett. 87, 047201 (2001).

[19] F. Merz and J. T. Chalker, Phys. Rev. B 65, 054425 (2002).

[20] F. Merz and J. T. Chalker, Phys. Rev. B 66, 054413 (2002).

[21] S. L. A. de Queiroz and R. B. Stinchcombe, Phys. Rev. B 68, 144414 (2003).

[22] S. L. A. de Queiroz, Phys. Rev. B 73, 064410 (2006).

[23] J. L. Cardy, J. Phys. A 19, L1093 (1986).

[24] A. W. W. Ludwig and J. L. Cardy, Nucl. Phys. B 285, 687 (1987). 